Annuaire suisse de politique de développement

25-2 | 2006

Paix et sécurité : les défis lancés à la coopération internationale

\title{
En guise d'introduction : objectifs et contenu du dossier
}

Xavier Tschumi Canosa et Thania Paffenholz

\section{(2) OpenEdition \\ Journals}

Édition électronique

URL : http://journals.openedition.org/aspd/238

DOI : 10.4000/aspd.238

ISSN : 1663-9669

Éditeur

Institut de hautes études internationales et du développement

Édition imprimée

Date de publication : 1 octobre 2006

Pagination : 11-16

ISBN : 2-88247-064-9

ISSN : $1660-5934$

Référence électronique

Xavier Tschumi Canosa et Thania Paffenholz, « En guise d'introduction : objectifs et contenu du dossier », Annuaire suisse de politique de développement [En ligne], 25-2 | 2006, mis en ligne le 18 décembre 2009, consulté le 21 septembre 2020. URL : http://journals.openedition.org/aspd/238 ; DOI : https://doi.org/10.4000/aspd.238 


\title{
En guise d'introduction: objectifs et contenu du dossier
}

\author{
Xavier Tschumi Canosa* et Thania Paffenholz**
}

Objectifs du dossier

Le présent dossier est consacré à la guerre et à la paix, questions qui ont toujours tourmenté l'humanité. L'Annuaire suisse de politique de développement s'efforce d'élucider de plus près les rapports entre paix, sécurité et développement. Différentes raisons nous paraissent justifier l'étude de ce thème hic et nunc.

Depuis une bonne décennie, le thème «paix, sécurité et développement» s'est installé plus fermement dans la coopération internationale. Depuis la fin du conflit Est-Ouest, la diplomatie classique se préoccupe davantage de la prévention et du règlement des conflits armés et des guerres au sein même des Etats; la coopération au développement et l'aide humanitaire en discutent depuis la tragédie du génocide rwandais de 1994.

La plupart des pays connaissant des situations de conflits internes ou régionaux sont des pays en développement et, depuis la fin de la guerre froide, pour leur venir en aide, les pays traditionnellement donneurs d'aide ont intégré une composante de politique de paix dans leur politique de coopération au développement. Bien que localisés dans les pays en développement, ces conflits n'en ont pas moins des conséquences régionales et même globales, notamment par rapport aux ressources naturelles stratégiques ou en ce qui concerne les flux migratoires.

Dans cette dynamique, de nouveaux concepts ont été introduits et opérationnalisés, tel celui de sécurité humaine, qui fait le lien entre le développement humain de l'individu et son intégrité physique. Des méthodes scientifiques d'analyse des conflits ont également été transformées en «outils» pratiques. Ici, la dimension économique des conflits entre dans les discussions. Il existe aussi entretemps une large palette d'instruments pour concevoir des projets de développement en tenant compte des conflits. La recherche théorique foisonne et porte sur les conditions qui sont à la base des conflits et de leur perpétuation ainsi que sur les conditions pour les terminer et promouvoir la paix. Toute une littérature explore aussi le défi extraordinaire consistant à prévenir les conflits. La plupart de ces recherches sont publiées en anglais et c'est une ambition du dossier de l'Annuaire que d'apporter ces éléments conceptuels et de réflexion dans les langues française et allemande.

En Suisse, depuis le tournant du millénaire, des agences étatiques et non étatiques se penchent de façon accrue sur les questions conceptuelles et institutionnelles

* Collaborateur scientifique à l'Institut universitaire d'études du développement (IUED), Genève, et responsable du présent dossier.

** Collaboratrice scientifique et de l'enseignement, IUED, Genève. 
liées à la promotion et au développement de la paix, et s'engagent par la diplomatie ou des opérations dans de nombreux pays en conflit. Fondée en 2000, la Division politique IV «Sécurité humaine» (DP IV) est devenue entre-temps la plus étoffée du ministère suisse des affaires étrangères (DFAE). Au sein de la Direction du développement et de la coopération (DDC), une nouvelle section a été fondée en 2001, qui se spécialise dans les questions de la paix et des conflits (COPRET); plusieurs organisations non gouvernementales (ONG) de développement ont ouvert des bureaux pour les conflits et développent leur engagement dans les pays en conflit. Fondé en 2001, le Centre pour la promotion de la paix (KOFF) de Swisspeace est un réseau qui unit les acteurs étatiques et non étatiques. Cette évolution fournit l'occasion de dresser un bilan de ce qui est discuté au niveau national ou international dans les sciences et la pratique, et de tirer les leçons des expériences faites.

\section{Contenu du dossier}

Le contenu du dossier reflète cette évolution. Les articles de la première partie donnent un aperçu des tendances et des expériences internationales, tandis que la deuxième aborde les conflits à propos des ressources, auxquels la Suisse s'est toujours intéressée et pour lesquels elle dispose d'une grande expertise. La troisième partie traite de la promotion et du développement de la paix tels que la Suisse les pratique et explique à propos de trois cas d'école (Népal, Colombie et Balkans) les chances et les gageures d'une politique suisse cohérente de sécurité, de paix et de développement.

\section{Coopération internationale au cœur des conflits: éclairages conceptuels}

La première partie du dossier donne tout d'abord à Thania Paffenholz l'occasion de définir les notions centrales de conflit et de promotion de la paix, dans une perspective historique et dans leur relation au développement. Outre sa volonté de poser les bases conceptuelles nécessaires à la compréhension de tout le dossier, elle analyse le rôle de promoteur de la paix que peut jouer la coopération internationale dans les pays en conflit. A l'issue de cet exercice, l'auteure préconise de ne plus confiner la coopération à une simple fonction instrumentale, mais de l'inscrire dans une vision stratégique et politique plus large, incluant notamment le renforcement des capacités dans les pays bénéficiaires.

Neclâ Tschirgi rend compte, dans son article, des résultats d'un programme de recherche qu'elle a dirigé, intitulé Security-Development Nexus. Sur la base de recherches empiriques dans divers pays, elle montre de manière convaincante que le lien entre les deux objectifs de sécurité et de développement est bien plus complexe que ne le laisse penser la rhétorique générale et que l'efficacité des politiques poursuivies pour les atteindre ne passe pas forcément par leur intégration mutuelle.

Dans la même lignée, mais sur une thématique plus spécifique, Roland Paris a examiné de nombreuses missions de consolidation de la paix menées par l'ONU, en se demandant si celles-ci, en promouvant comme modèles la démocratisation et la libéralisation des marchés, sont adaptées à la situation fragile des Etats récemment sortis d'un conflit. L'article présenté dans ce dossier est adapté 
d'une interview menée par Rocha Menocal et Kate Kilpatrick, parue en novembre 2005 dans Development in Practice. L'auteur analyse que ces modèles peuvent au contraire être sources de nouveaux conflits dans des pays n'ayant pas les prérequis nécessaires pour les absorber.

Ces trois articles montrent à l'évidence que la recherche académique a un rôle fondamental à jouer pour permettre de dénouer un peu l'écheveau des fils qui relient entre elles les notions de paix, de développement et de sécurité. Toutefois, dans le dernier article de cette première partie, Riccardo Bocco illustre avec pertinence que ce rôle n'est pas facile et que, malgré la qualité des recherches, un fossé peut exister entre leurs résultats et la décision politique qu'ils sont censés faciliter. Il se base pour cela sur le programme d'étude des conditions de vie socio-économiques de la population palestinienne qu'il conduit avec son équipe sur le terrain depuis 2000 et sur l'usage qui en est fait sur le plan politique, tant par les dirigeants palestiniens que par la communauté internationale.

\section{Liens entre conflits et ressources naturelles: une affaire entendue?}

Afin d'illustrer plus en détail les questions cruciales qui peuvent se poser dans les interrelations entre les notions de paix, de développement et de sécurité, la deuxième partie du dossier est consacrée à la problématique exemplaire des liens entre conflits violents et ressources naturelles. Ces dernières sont en effet au centre de nombreuses et diverses configurations dans lesquelles les objectifs de paix, de développement (durable) et de sécurité s'entrechoquent.

Laurent Goetschel et Didier Péclard rendent compte, dans leur contribution, des enseignements qu'ils ont pu tirer d'un projet individuel de recherche mené par la Fondation suisse pour la paix, projet qui s'inscrit dans un programme national de recherche intitulé NCCR North-South - Research Partnerships for Mitigating Syndromes of Global Change. Selon ces auteurs, la portée heuristique du lien de causalité supposé direct entre la diminution des ressources naturelles et la survenance de conflits violents doit être nuancée. Il y a en effet d'autres facteurs à prendre en compte, notamment historique, politique et économique, pour expliquer les conflits.

Dans son article, Claude Serfati prend, lui, le contre-pied des thèses avancées par la Banque mondiale sur les causes économiques des conflits, thèses qui établissent un lien de causalité entre l'existence de ressources naturelles dans un pays et les conflits armés qui peuvent s'y dérouler: les conflits auraient donc des causes endogènes locales et leurs impacts se ressentiraient mondialement en raison de l'imbrication croissante des économies. L'auteur, en étudiant dans son article la rente procurée par les ressources naturelles, tient pour vrai le lien de corrélation inverse : ce seraient plutôt les laissés-pour-compte de la mondialisation qui fournissent le ferment des guerres locales. Dans un très court article également lié à la question de la mondialisation, Gabi Hesselbein apporte un éclairage avec l'exemple de la République démocratique du Congo. L'article montre que, malgré les richesses naturelles dont jouit le pays, celui-ci peine à sortir de la spirale d'instabilité politique et de conflits qui l'affecte depuis son indépendance en 1960.

L'essai de Wolfgang Sachs, qui termine la deuxième partie du dossier, utilise l'exemple du pétrole pour mettre en évidence les conflits de survie, de régime, de 
répartition et de développement (durable) que peut provoquer cette ressource naturelle particulière. L'auteur conclut qu'il n'est pas satisfaisant d'étudier les conflits pour les ressources sous l'angle uniquement de la sécurité, mais qu'il faut introduire dans l'analyse la notion de justice, dont l'œuvre conduit à la paix.

\section{Acteurs suisses: quelles contributions et quelles ambitions?}

Dans cette troisième partie, il s'agit de décrire et d'analyser comment la nouvelle donne mondiale depuis le début des années 1990 a influencé les acteurs suisses, gouvernementaux et non gouvernementaux, actifs dans la thématique du présent dossier. Cette focalisation sur la Suisse permet de quitter le niveau des concepts et des idées pour se concentrer, cette fois, sur les orientations et la mise en œuvre des politiques de paix, de développement et de sécurité.

En introduction à cette partie du dossier, Xavier Tschumi Canosa présente comment, dans le Rapport sur la politique extérieure 2000 de la Suisse, l'objectif visant à la coexistence pacifique des peuples a permis de façonner la politique de sécurité et de paix de ce pays et l'a orientée vers la prévention des conflits et la promotion de la paix. Pour cela, l'auteur se base, d'une part, sur les documents officiels attestant un tournant de la politique extérieure et, d'autre part, sur une interview effectuée avec trois responsables de l'administration fédérale impliqués de près dans la définition et la mise en œuvre de la politique de promotion de la paix.

Dans son article, Maya Krell décrit le rôle que jouent les ONG suisses dans la dynamique d'adaptation de la coopération au développement aux contraintes imposées par les situations de conflits dans lesquelles elle est mise en œuvre. Cette contribution met en relief la tension qui peut exister entre, d'une part, la prise en compte des conflits (conflict sensitive) dans la coopération au développement et, d'autre part, la promotion des droits humains que celle-ci a endossée. L'article, qui décrit et documente la problématique, expose également les processus d'apprentissage engagés par les ONG ainsi que les défis auxquels elles sont confrontées et les possibilités qui s'offrent à elles.

Les articles qui complètent cette partie du dossier montrent par des exemples comment, concrètement, la Suisse construit le lien entre paix et sécurité d'une part et coopération internationale d'autre part.

Le premier exemple, signé par Günther Baechler et Jörg Frieden, concerne le Népal. Il présente la stratégie actuelle de la Suisse dans ce pays, articulant d'un côté les programmes de coopération au développement et, de l'autre, les activités de promotion de la paix et des droits humains. Pour ces dernières, la Suisse capitalise clairement ses décennies de coopération au développement avec le Népal et notamment la confiance créée à cette occasion. L'article montre en quoi «le Népal est ainsi devenu pour la Suisse un test concret de cohérence dans la mise en œuvre complémentaire et cumulative des divers instruments de politique étrangère».

Le deuxième exemple porte sur le processus de paix en Colombie, auquel la Suisse est partie prenante. L'article de Cristina Hoyos explore les avantages comparatifs sur lesquels la Suisse a pu compter pour prendre ce rôle de médiateur-facilitateur. Il évoque en particulier son engagement humanitaire et neutre dans le pays ainsi que, et c'est lié, les rapports de confiance solides qu'elle a 
patiemment su construire avec toutes les parties au conflit. Par un court éclairage, Jean-Pierre Gontard illustre ce propos en expliquant le rôle pionnier de l'Institut universitaire d'études du développement (IUED) dans l'engagement de la Suisse en Colombie. Ces deux contributions, volontairement circonspectes sur les détails des négociations en cours, trahissent, il est vrai, la nature délicate d'une telle action diplomatique. Mais, à l'instar de l'exemple népalais, elles mettent en évidence en quoi la présence préalable de longue durée de la Suisse en Colombie a permis cette action.

Le dernier exemple concerne la région des Balkans, dans laquelle la Suisse est impliquée à plusieurs niveaux. Andreas Ernst, profitant de son regard de journaliste, relaie la parole d'acteurs suisses de la coopération au développement présents sur place. Dans sa conclusion, il commente la prise de position de la conseillère fédérale Micheline Calmy-Rey sur l'indépendance du Kosovo et se fait l'écho des frustrations que cette prise de position a engendrées parmi les coopérants suisses travaillant dans cette province. On perçoit ainsi de manière palpable comment un engagement suisse qui se déroule simultanément au niveau macropolitique et au niveau opérationnel peut causer un problème classique de cohérence. René Holenstein, dans un court article, présente quant à lui le projet «Plate-forme Bosnie-Herzégovine», lancé en 2005 par la DDC. Avec cette contribution, l'auteur montre l'importance du dialogue entre les différentes minorités en vue d'une unité économique, sociale et politique du pays, et comment la coopération internationale de la Suisse peut contribuer à cet objectif.

\section{Résumé et perspectives}

Le présent dossier ne donne pas seulement une bonne vue d'ensemble des rapports entre paix, sécurité et coopération internationale, mais met également en lumière la position des acteurs suisses et la manière dont ceux-ci voient leur rôle. Nous avons fait les constats suivants.

L'approche scientifique de la paix et de la sécurité dans la coopération internationale se divise en deux tendances fondamentales. D'une part, on réfléchit beaucoup à des questions de détail comme une préparation des projets de développement qui tienne compte des conflits dans leur contexte local, ou les meilleurs modèles de désarmement et de réinsertion des anciens combattants dans leur communauté. D'autres études scientifiques se penchent sur les problèmes nationaux et mondiaux de la sécurité, de la paix et de la coopération internationale. On citera à titre d'exemple la discussion concernant le «bon» régime économique et gouvernemental pour les pays qui sortent d'un conflit (voir l'article de Roland Paris) ou le débat sur les effets globaux et les intérêts occidentaux liés aux économies de guerre (Claude Serfati).

Chose intéressante, la pratique se réfère souvent au premier discours, plus «technique», alors que l'on discute moins les questions nationales et mondiales de fond. Ainsi, le débat sur la démarche do no harm a connu une forte notoriété dans les projets de développement, alors que ses conséquences politiques ont été peu intégrées dans le travail de terrain, même si elles ont été perçues à la marge.

Les cas d'école de la Colombie et du Népal montrent cependant nettement l'importance de la dimension politique de la coopération internationale pour instaurer la paix et la sécurité dans un pays. Il s'agit d'une part de la concertation 
entre les interventions des diplomates et celles de la politique de développement. De l'autre, des cas comme le Népal montrent que, dans le concert des donateurs, la dimension politique du développement est nécessaire pour donner des impulsions vitales à la paix et à la démocratie. La Suisse est justement un acteur prédestiné du fait que les différents acteurs suisses peuvent intervenir à tous les niveaux. Mais sans une stratégie suisse cohérente, qui exerce aussi sciemment son influence sur les autres, l'action suisse sera fortement limitée. Il ne suffit toutefois pas d'établir des synergies entre les projets de terrain et le travail politique; il faut plutôt définir un ordre de priorité clair, qui dise quel genre de projets peut contribuer le mieux à améliorer l'efficacité du travail politique.

En fin de compte, un principe doit rester évident: la paix et la sécurité peuvent certes être promues de l'extérieur, par des moyens diplomatiques, militaires ou de la politique de développement, mais la propriété (ownership) doit en rester aux acteurs locaux.

Au nom du comité de rédaction de l'Annuaire suisse de politique de développement, nous voudrions remercier toutes les personnes qui ont contribué, de près ou de loin, à l'élaboration du présent dossier.

Nous pensons d'abord à tous les auteurs qui ont apporté leur contribution à cet ouvrage et qui ont patiemment aménagé leurs textes de telle façon qu'ils se répondent les uns aux autres et qu'ils constituent ensemble, in fine, un tout cohérent et riche. Puissent leurs efforts trouver dans ce numéro leur récompense.

Nous tenons ensuite à remercier officiellement la Division politique IV (DFAE) ainsi que les sections Politique de développement et COPRET de la DDC (DFAE) d'avoir soutenu financièrement le présent dossier. Nous gageons que celui-ci corroborera leurs attentes et qu'il trouvera bonne place dans les bibliothèques des offices fédéraux actifs dans les domaines de la paix, du développement et de la sécurité.

Nous voudrions enfin sincèrement remercier les personnes qui, en septembre 2005, ont participé à notre séance de réflexion initiale et qui, par leurs apports et leur réseau de connaissances, ont permis de structurer le dossier et de faciliter l'identification et le contact des auteurs potentiels. Il s'agit d'Anita Müller, directrice de projet au Centre pour la promotion de la paix (KOFF), de Peter Niggli, directeur d'Alliance Sud, et de Riccardo Bocco, professeur ordinaire à l'IUED. Nous remercions particulièrement Guillaume Scheurer, chef adjoint de la Division politique IV, pour sa participation à cette séance mais aussi pour son aide précieuse et sa disponibilité tout au long du processus d'élaboration de ce dossier ainsi que Martin Fässler, chef de la section Politique de développement de la DDC, pour sa contribution lors de la séance et plus largement pour le dossier.

Pour leur implication dans ce dossier et leurs encouragements, notre gratitude va à l'ambassadeur Thomas Greminger, chef de la Division politique IV, à l'ambassadeur Raimund Kunz, chef de la Direction de la politique de sécurité au sein du DDPS, ainsi qu'à Cristina Hoyos, cheffe de la section COPRET de la DDC.

Nos remerciements chaleureux s'adressent encore à Jacques Forster, professeur à l'IUED, qui a conduit avec autorité cette séance de réflexion avant de quitter son poste de directeur de notre comité, ainsi qu'à Christoph Stamm, membre du comité jusqu'en janvier 2006, sans l'apport duquel le dossier ne serait pas parti sur d'aussi bons rails. 